\title{
Analysis of posterior circulation diameters depending on age, sex and side by computed tomography angiography
}

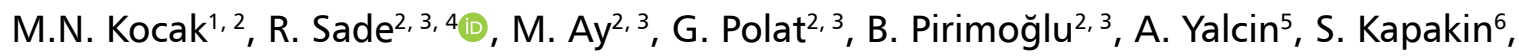 \\ I.M. Kabakus ${ }^{7}, \mathrm{M} . \mathrm{Ur}^{8}$ \\ ${ }^{1}$ Department of Neurology, Medical Faculty, Ataturk University, Erzurum, Turkey \\ 2Educational and Training Hospital, Ataturk University, Erzurum, Turkey \\ ${ }^{3}$ Department of Radiology, Medical Faculty, Ataturk University, Erzurum, Turkey \\ ${ }^{4}$ Clinical Research, Development and Design Application and Research Centre, Ataturk University, Erzurum, Turkey \\ ${ }^{5}$ Department of Radiology, Medical Faculty, Binali Yildirim University, Erzincan, Turkey \\ ${ }^{6}$ Department of Anatomy, Medical Faculty, Ataturk University, Erzurum, Turkey \\ ${ }^{7}$ Department of Radiology and Radiological Science, Medical University of South Carolina, South Carolina, United States \\ ${ }^{8}$ Siemens Healthcare, Istanbul, Turkey
}

[Received: 18 June 2020; Accepted: 20 July 2020; Early publication date: 7 August 2020]

Background: Posterior circulation of brain is important because of vital organs' blood supply provided by them. In this study, we evaluate the relationship of posterior circulation measurements with age, gender and side by using computed tomography angiography (CTA) images.

Materials and methods: A total 199 brain CTA examinations were retrospectively analysed for all posterior circulation arteries (vertebral artery, basilar artery, posterior cerebral artery [PCA], superior cerebellar artery [SCA], anterior inferior cerebellar artery, and posterior inferior cerebellar artery [PICA]) to compare the difference based on age, gender and side.

Results: There is no correlation between age and the mean diameters of all vessels ( $p>0.05)$. The mean diameter of left vertebral artery was higher than right vertebral artery in all genders ( $p=0.004$ for males and $p<0.001$ for females). The mean diameter of left SCA and PICA were higher than right SCA and PICA in females ( $p=0.032$ and $p=0.027$, respectively). The mean diameters of basilar, left $P C A$, left SCA, left vertebral, right PCA, right SCA, right PICA and right vertebral artery were higher in males and that differences were statistically significant $(p<0.001, p=0.002, p=0.006, p=0.004, p=0.001, p=0.003, p=0.002$, and $p=0.006$, respectively).

Conclusions: The posterior circulation vessel diameter is not affected by aging. The mean diameters of basilar artery, both PCAs, both SCAs, right PICA, both vertebral arteries were higher in males. The mean diameter of left vertebral artery is higher than that of right vertebral artery in all genders. (Folia Morphol 2021; 80, 3: 527-532)

Key words: cerebral arteries, cerebellar arteries, posterior circulation, morphometric measurements, computed tomography 


\section{INTRODUCTION}

Posterior circulation consists of vertebral, basilar, posterior cerebral, superior cerebellar, anterior inferior cerebellar and posterior inferior cerebellar arteries, as well as their branches. It is very variable and sometimes complex. Posterior circulation vascularizes the posterior part of the brain, in which many vital structures, such as the cerebellum, thalamus, and brainstem, are located. Although digital subtraction angiography (DSA) is the gold standard in vascular imaging, it has been shown that computed tomography angiography (CTA) has become as effective as DSA with the advances in technology [2]. A brain CTA examination is a non-invasive evaluation method. There are several morphologic studies which evaluate the brain arterial system $[2,6,8,12]$. There are several studies investigating the variations in the posterior circulation system $[2,6,11]$; however, those involving the measurement of arterial diameter are limited in number $[8,13]$. To our knowledge, the current study is the first in English language literature that explored the relationship between posterior circulation arteries and age, gender, and side simultaneously.

\section{MATERIALS AND METHODS}

This retrospective study included the data from the patient files gathered from the local picture archiving and communication systems between January 2019 and January 2020. A total of 256 brain CTA examinations were included. CTA examinations were performed on a 320-row detector computed tomography (CT) (Aquillion ONE Vision; Toshiba Medical Systems Corporation, Otawara, Japan), or a 256-row detector CT (Somatom ${ }^{\circledR}$ Definition Flash, Siemens Healthcare, Forchheim, Germany). The CT acquisition protocol was also performed with the following parameters: 0.5 -s gantry rotation time, $0.5 \mathrm{~mm}$ slice thickness, $128 \times$ $\times 0.6-\mathrm{mm}$ or $192 \times 0.6-\mathrm{mm}$ collimation using a z-flying focal spot, $200 \mathrm{mAs}$ tube current at $120 \mathrm{kVp}$ tube voltage. For optimal intraluminal contrast enhancement, the delay time between start of contrast material administration and start of scanning was determined for each patient individually by using a bolus-tracking technique. A total of $60-75 \mathrm{~mL}$ iopromide (Ultravist 370 mg/mL, Bayer Schering Pharma, Berlin, Germany), an automatic injector was used (MCT Plus; Medrad, Pittsburgh, PA) over $15 \mathrm{~s}$ through an 18-gauge intravenous line placed into the right antecubital vein at a rate of $4-5 \mathrm{~mL} / \mathrm{s}$. The contrast produced a sensation of "hot flash". Immediately following the injection of the iodinated contrast, $50 \mathrm{~mL}$ saline was infused by the same injector via the same route.

The cases with trauma, tumour or vascular pathologies, paediatric cases, and repetitive examinations were excluded (Fig. 1). As a result, a total of 199 brain CTA examinations were evaluated on axial, coronal, or sagittal images by 2 radiologists with 3 and 10 years of neuroradiology experience. The diameter of the vertebral artery (VA) was measured on the intracranial segment (V4), $1 \mathrm{~cm}$ before the confluence. The diameters of the posterior cerebral artery (PCA) were measured on the P1 segment. If there was no P1 or V4 segment, the measurement was not performed. The diameter of the basilar artery (BA) was measured from the mid-part. The diameters of the superior cerebellar artery (SCA), anterior inferior cerebellar artery (AICA), and posterior inferior cerebellar artery (PICA) were measured from the proximal part (Fig. 2). The measurements and patients' demographic data were recorded.

\section{Statistical analysis}

Statistical analyses were performed using SPSS v. 22.0 (SPSS Inc., Chicago IL, USA). The suitability of the data for normal distribution was evaluated by the single-sample Kolmogorov-Smirnov test. Levene's statistics were used for the homogeneity analysis of group variances. The independent-samples t-test was conducted to determine the differences between male and female biometric measurements, and the paired-samples t-test was utilised to determine those between the left and right measurements of men and women. The significance level was accepted as $p<0.05$.

\section{RESULTS}

The mean age of the patients was $48.55 \pm 15.82$ (range 18-91) years. Of the patients, 105 (52.76\%) were female. The mean diameters were calculated as $3.34 \pm 0.59$ (range 1.35-5.3) $\mathrm{mm}$ for BA, $1.79 \pm$ \pm 0.47 (range 0.52-3.50) $\mathrm{mm}$ for the left PCA, $1.12 \pm$ \pm 0.33 (range 0.40-2.18) $\mathrm{mm}$ for the left SCA, $1.02 \pm$ \pm 0.34 (range 0.33-1.98) $\mathrm{mm}$ for the left AICA, $1.26 \pm$ \pm 0.36 (range 0.40-2.20) $\mathrm{mm}$ for the left PICA, $2.99 \pm$ \pm 0.70 (range 0.95-5.13) $\mathrm{mm}$ for the left VA, $1.76 \pm$ \pm 0.47 (range 0.80-2.94) $\mathrm{mm}$ for the right PCA, $1.06 \pm$ \pm 0.31 (range 0.36-2.10) $\mathrm{mm}$ for the right SCA, $0.95 \pm$ \pm 0.31 (range 0.30-1.76) $\mathrm{mm}$ for the right AICA, $1.18 \pm$ \pm 0.36 (range $0.43-2.56$ ) $\mathrm{mm}$ for the right PICA, and $2.68 \pm 0.71$ (range $0.94-4.52$ ) $\mathrm{mm}$ for the right VA. 


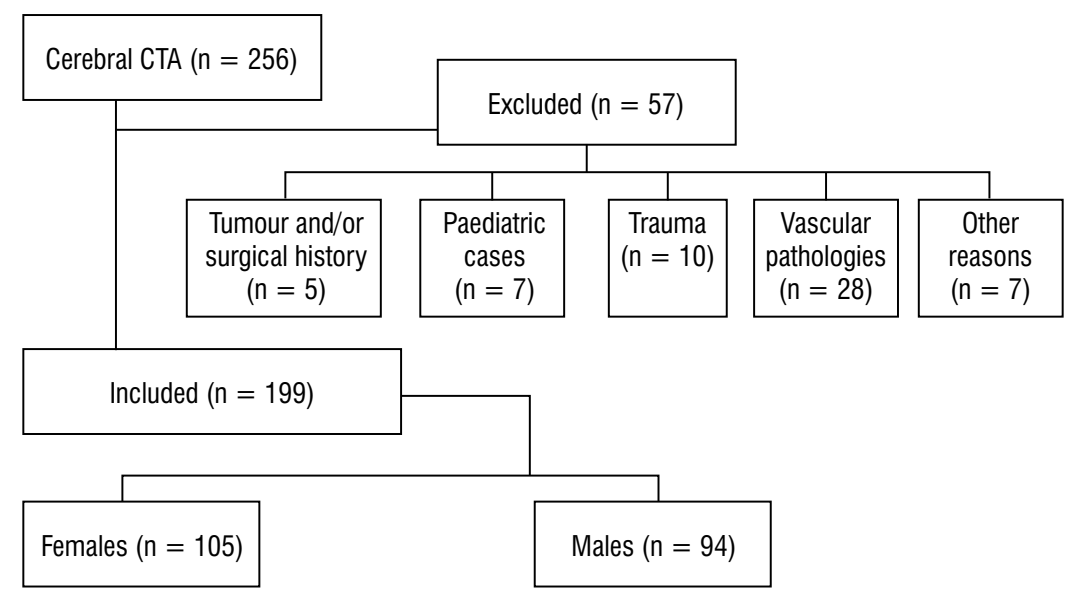

Figure 1. Study flow diagram; CTA — computed tomography angiography.

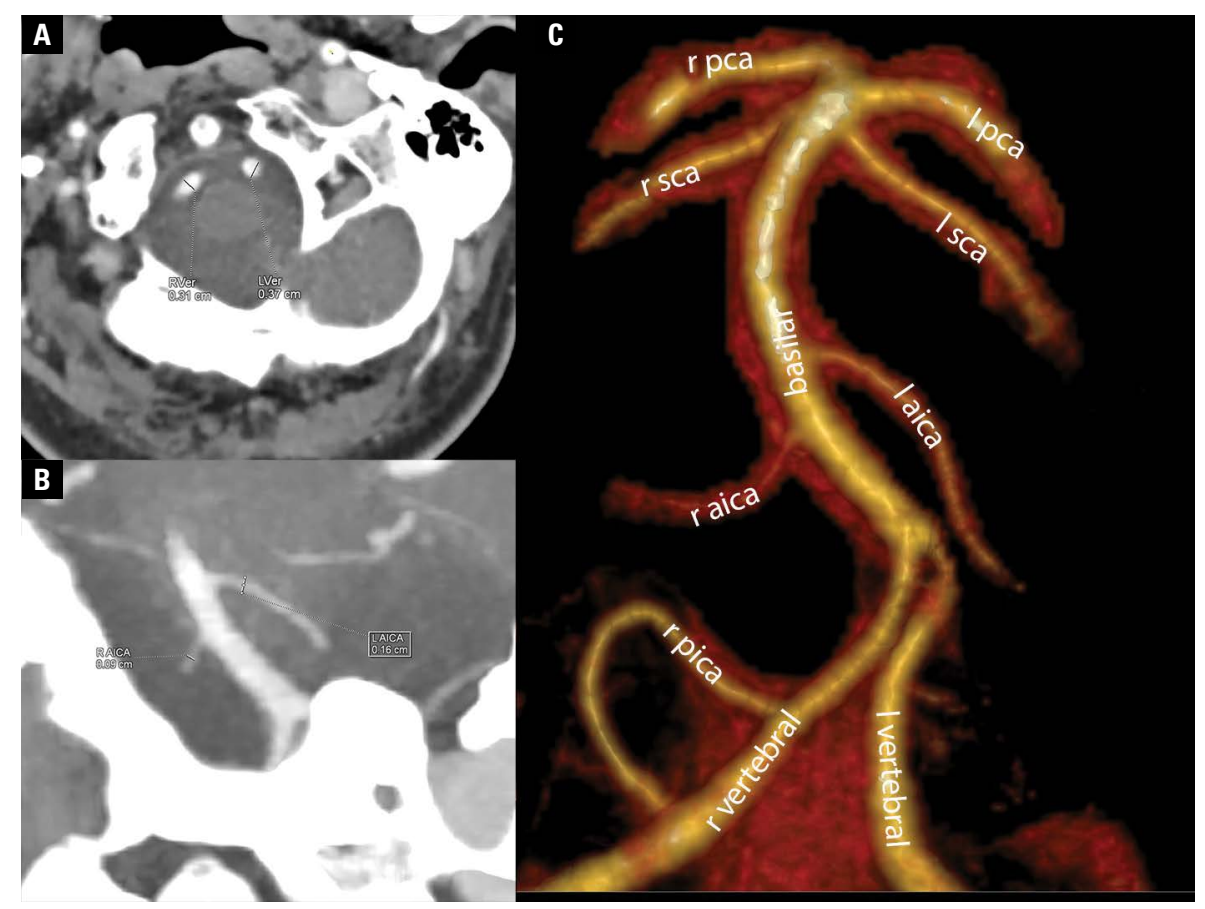

Figure 2. Axial (A), coronal (B) and three-dimensional volume rendered (C) computed tomography images of a 51-year-old male. The measurements of right vertebral artery ( $r$ vertebral) and left vertebral artery (I vertebral) are shown on axial image (B). The measurements of right anterior inferior cerebral artery ( $r$ aica) and left anterior inferior cerebral artery (l aica) are shown on axial image; $r$ pica — right posterior inferior cerebellar artery; I pca — left posterior cerebral artery; r pca — right posterior cerebral artery; I sca — left superior cerebellar artery; r sca - right superior cerebellar artery.

There was no correlation between age and the mean diameters of any of the arteries $(p>0.05)$

The mean diameters of BA, left PCA, left SCA, left VA, right PCA, right SCA, right PICA, and right VA were statistically significantly higher in males than in females (Table 1). Left foetal-type PCA was observed in 4 males and 5 females. Right foetal-type PCA was detected in 2 males and 2 females. There was no statistically significant difference in foetal-type PCA variations between the genders $(p=0.95)$. The left SCA could not be visualised in 1 male, the right SCA in 1 female, the left AICA in 34 males and 39 females, the right AICA in 30 males and 33 females, the left PICA in 2 males and 5 females, the right PICA in 
Table 1. The intergroup comparison of the posterior circulation arteries measurements by gender

\begin{tabular}{|c|c|c|c|c|c|c|c|}
\hline Artery & Gender & Number & Mean & Standard deviation & $\mathbf{t}$ & df & $\mathbf{P}$ \\
\hline \multirow[t]{2}{*}{ Basilar } & Male & 94 & 3.52 & 0.61 & 4.033 & 197 & 0.000 \\
\hline & Female & 105 & 3.18 & 0.58 & & & \\
\hline \multirow[t]{2}{*}{ Left PCA } & Male & 90 & 1.91 & 0.47 & 3.195 & 188 & 0.002 \\
\hline & Female & 100 & 1.68 & 0.48 & & & \\
\hline \multirow[t]{2}{*}{ Left SCA } & Male & 93 & 1.18 & 0.35 & 2.768 & 196 & 0.006 \\
\hline & Female & 105 & 1.06 & 0.29 & & & \\
\hline \multirow[t]{2}{*}{ Left AICA } & Male & 60 & 1.01 & 0.37 & -0.155 & 124 & 0.877 \\
\hline & Female & 66 & 1.02 & 0.3 & & & \\
\hline \multirow[t]{2}{*}{ Left PICA } & Male & 92 & 1.30 & 0.37 & 1.520 & 190 & 0.130 \\
\hline & Female & 100 & 1.22 & 0.35 & & & \\
\hline \multirow[t]{2}{*}{ Left vertebral } & Male & 94 & 3.14 & 0.75 & 2.924 & 197 & 0.004 \\
\hline & Female & 105 & 2.85 & 0.62 & & & \\
\hline \multirow[t]{2}{*}{ Right PCA } & Male & 92 & 1.88 & 0.49 & 3.514 & 193 & 0.001 \\
\hline & Female & 103 & 1.65 & 0.43 & & & \\
\hline \multirow[t]{2}{*}{ Right SCA } & Male & 94 & 1.13 & 0.35 & 2.965 & 167 & 0.003 \\
\hline & Female & 104 & 0.99 & 0.25 & & & \\
\hline \multirow[t]{2}{*}{ Right AICA } & Male & 64 & 0.95 & 0.31 & -0.137 & 134 & 0.892 \\
\hline & Female & 72 & 0.96 & 0.31 & & & \\
\hline \multirow[t]{2}{*}{ Right PICA } & Male & 92 & 1.26 & 0.38 & 3.065 & 187 & 0.002 \\
\hline & Female & 97 & 1.10 & 0.32 & & & \\
\hline \multirow[t]{2}{*}{ Right vertebral } & Male & 94 & 2.82 & 0.7 & 2.765 & 195 & 0.006 \\
\hline & Female & 103 & 2.55 & 0.69 & & & \\
\hline
\end{tabular}

PCA — posterior cerebral artery; SCA — superior cerebellar artery; AICA — anterior inferior cerebellar artery; PICA — posterior inferior cerebellar artery

2 males and 8 females, and the V4 segment of the right VA in 2 females.

The mean diameters of SCA, PICA, and VA were statistically significantly higher on the left side compared to the right side (Table 2). However, in gender-based subgroup analyses, a statistically significant result was observed in only 1 artery (VA) in both genders (Table 3 ). The mean diameters of SCA and PICA were higher on the left side in both males and females but the differences were not statistically significant in males (Table 3). The mean diameter of the left VA was higher than that of the right VA in both genders.

\section{DISCUSSION}

In this study, a total of 13 foetal-type PCA variations $(6.53 \%)$ were observed, which is a lower percentage than reported in the literature $[6,11]$. Han et al. [2] showed that CTA with 1-mm slice thickness underestimated cerebral arteries compared to DSA. In the current study, we used $0.5-\mathrm{mm}$ slice thickness for CTA. Thus, we consider that our different results were due to our $\mathrm{CT}$ device being able to show smaller vessels. This is supported by similar results obtained from cadaver studies [9, 13]. Another explanation may be that there is a difference in the rate of variation in different societies. Further thin-slice CTA and cadaver studies on this subject can provide a better explanation.

In this study, the diameters of VA, BA, PCA, PICA and AICA were similar to the ranges in the literature (2.8 $\mathrm{mm}$ vs. $2.2-2.8 \mathrm{~mm}, 3.3 \mathrm{~mm}$ vs. $2.7-3.6 \mathrm{~mm}$, $1.8 \mathrm{~mm}$ vs. $1.6-2.2 \mathrm{~mm}, 1.2 \mathrm{~mm}$ vs. $1.2-1.7 \mathrm{~mm}$, and $1 \mathrm{~mm}$ vs. $1 \mathrm{~mm}$, respectively) [4, 7, 8, 10, 12, 13]. However, the SCA diameter was lower than the literature range $(1.1 \mathrm{~mm}$ vs. $1.3-1.4 \mathrm{~mm})[1,5,7]$. This could be related to the differences in the method (cadaver versus CTA), technique $(1 \mathrm{~mm}$ vs. $0.5 \mathrm{~mm}$ slice thickness), or patient selection (inclusion and exclusion criteria).

Rai et al. [8] revealed that the vessel calibre was affected by age. However, Ichikawa et al. [3] found no correlation between age and vessel calibre. Furthermore Vitosevic et al. [12] showed that the calibre of BA was higher in the elderly but those of VA and 
Table 2. The intergroup comparison of the posterior circulation arteries measurements by side

\begin{tabular}{|c|c|c|c|c|c|c|c|}
\hline Artery & Side & Number & Mean & Standard deviation & $t$ & df & $\mathbf{P}$ \\
\hline \multirow[t]{2}{*}{ PCA } & Right & 186 & 1.76 & 0.47 & -0.624 & 185 & 0.533 \\
\hline & Left & 186 & 1.78 & 0.49 & & & \\
\hline \multirow[t]{2}{*}{ SCA } & Right & 197 & 1.06 & 0.31 & -2.692 & 196 & 0.008 \\
\hline & Left & 197 & 1.12 & 0.33 & & & \\
\hline \multirow[t]{2}{*}{ AICA } & Right & 120 & 0.95 & 0.31 & -1.657 & 119 & 0.100 \\
\hline & Left & 120 & 1 & 0.32 & & & \\
\hline \multirow[t]{2}{*}{ PICA } & Right & 187 & 1.18 & 0.36 & -2.274 & 186 & 0.024 \\
\hline & Left & 187 & 1.25 & 0.36 & & & \\
\hline \multirow[t]{2}{*}{ Vertebral } & Right & 197 & 2.68 & 0.71 & -4.816 & 196 & 0.000 \\
\hline & Left & 197 & 2.9 & 0.7 & & & \\
\hline
\end{tabular}

PCA — posterior cerebral artery; SCA — superior cerebellar artery; AICA — anterior inferior cerebellar artery; PICA — posterior inferior cerebellar artery

Table 3. The subgroup comparison of the posterior circulation arteries measurements by gender

\begin{tabular}{|c|c|c|c|c|c|c|c|}
\hline Artery & Side & Number & Mean & Standard deviation & $t$ & df & $\mathbf{P}$ \\
\hline \multicolumn{8}{|l|}{ Male } \\
\hline \multirow[t]{2}{*}{ PCA } & Right & 88 & 1.86 & 0.49 & -0.672 & 87 & 0.503 \\
\hline & Left & 88 & 1.9 & 0.47 & & & \\
\hline \multirow[t]{2}{*}{ SCA } & Right & 93 & 1.13 & 0.35 & -1.619 & 92 & 0.109 \\
\hline & Left & 93 & 1.18 & 0.35 & & & \\
\hline \multirow[t]{2}{*}{ AICA } & Right & 56 & 0.95 & 0.31 & -0.934 & 55 & 0.354 \\
\hline & Left & 56 & 0.99 & 0.35 & & & \\
\hline \multirow[t]{2}{*}{ PICA } & Right & 91 & 1.26 & 0.38 & -0.939 & 90 & 0.350 \\
\hline & Left & 91 & 1.30 & 0.37 & & & \\
\hline \multirow[t]{2}{*}{ Vertebral } & Right & 94 & 2.82 & 0.7 & -2.973 & 93 & 0.004 \\
\hline & Left & 94 & 3.14 & 0.75 & & & \\
\hline \multicolumn{8}{|l|}{ Female } \\
\hline \multirow[t]{2}{*}{ PCA } & Right & 98 & 1.66 & 0.43 & -0.192 & 97 & 0.848 \\
\hline & Left & 98 & 1.67 & 0.48 & & & \\
\hline \multirow[t]{2}{*}{ SCA } & Right & 104 & 0.99 & 0.25 & -2.168 & 103 & 0.032 \\
\hline & Left & 104 & 1.06 & 0.29 & & & \\
\hline \multirow[t]{2}{*}{ AICA } & Right & 64 & 0.95 & 0.32 & -1.393 & 63 & 0.169 \\
\hline & Left & 64 & 1 & 0.31 & & & \\
\hline \multirow[t]{2}{*}{ PICA } & Right & 96 & 1.1 & 0.32 & -2.242 & 95 & 0.027 \\
\hline & Left & 96 & 1.2 & 0.34 & & & \\
\hline \multirow[t]{2}{*}{ Vertebral } & Right & 103 & 2.55 & 0.69 & -3.962 & 102 & 0.000 \\
\hline & Left & 103 & 2.86 & 0.63 & & & \\
\hline
\end{tabular}

PCA — posterior cerebral artery; SCA — superior cerebellar artery; AICA — anterior inferior cerebellar artery; PICA — posterior inferior cerebellar artery

PCA were similar. In the current study, we observed no correlation between age and vessel calibre.

In this study, the mean diameters of BA, left PCA, right PCA, left SCA, right SCA, right PICA, left VA and right $V A$ were higher in males. Rai et al. and Ichikawa et al. revealed similar results in that the mean diameters of BA and VA were higher in males $[3,8]$. On the other hand, Vitosevic et al. [12] showed no diameter difference in $B A$ by gender. However, the authors did not evaluate SCA, AICA, and PICA; therefore, we could not compare our results.

In this study, the mean diameters of SCA, PICA and VA were higher on the left side. Vitosevic et al. showed no statistically significant diameter dif- 
ference in VA and PCA depending on the side [12]. However, several studies including the current study showed that the left VA diameter was higher than the right VA diameter $[4,7,8,10,13]$. Shrontz et al. [10] revealed that there was no diameter difference between the left and right sides for PCA, PICA, and AICA. Pai et al. [7] reported that the diameters of the left AICA and SCA were higher than those of the right side while two other studies showed no side-based diameter differences in SCA [1,5]. The current study has both similarities and differences compared to the literature, which can be attributed to the differences in the technique used, number of patients evaluated, and the anatomical variations between the samples.

\section{Limitations of the study}

There are several limitations of this study. First, a CTA study cannot provide as comprehensive data as a cadaveric study. Second, we used the slice thickness as $0.5 \mathrm{~mm}$; thus, we were not able to evaluate vessels that were smaller than $0.5 \mathrm{~mm}$ in diameter; however, $0.5 \mathrm{~mm}$ is the lowest available cross-sectional thickness of devices in current medical use. Another limitation concerns the small sample size. Finally, we excluded patients with vascular diseases, which may have affected our age-related evaluation.

\section{CONCLUSIONS}

A CTA examination is a valuable technique for vascular evaluation even in small vessels, such as PICA and AICA. The posterior circulation vessel diameter was not affected by normal aging. The mean diameters of the left VA were found to be higher than those of the right. Lastly, the mean diameters of BA, left PCA, right PCA, left SCA, right SCA, right PICA, left $V A$ and right $V A$ were higher in males compared to females.

\section{Conflict of interest: None declared}

\section{REFERENCES}

1. Garcia-Gonzalez U, Cavalcanti DD, Agrawal A, et al. Anatomical study on the "perforator-free zone": reconsidering the proximal superior cerebellar artery and basilar artery perforators. Neurosurgery. 2012; 70(3): 764-772, doi: 10.1227/ NEU.0b013e3182351f8e, indexed in Pubmed: 21904251.
2. Han A, Yoon DY, Chang SKi, et al. Accuracy of CT angiography in the assessment of the circle of Willis: comparison of volume-rendered images and digital subtraction angiography. Acta Radiol. 2011; 52(8): 889-893, doi: 10.1258/ ar.2011.110223, indexed in Pubmed: 21828003.

3. Ichikawa $\mathrm{H}$, Mukai M, Takahashi N, et al. Dilative arterial remodeling of the brain with different effects on the anterior and posterior circulation: an MRI study. J Neurol Sci. 2009; 287(1-2): 236-240, doi: 10.1016/j.jns.2009.06.029, indexed in Pubmed: 19695577.

4. Karatas A, Yilmaz H, Coban G, et al. The anatomy of circulus arteriosus cerebri (circle of willis): a study in turkish population. Turk Neurosurg. 2016; 26(1): 54-61, doi: 10.5137/1019-5149.JTN.13281-14.1, indexed in Pubmed: 26768869.

5. Krzyżewski RM, Stachura MK, Stachura AM, et al. Variations and morphometric analysis of the proximal segment of the superior cerebellar artery. Neurol Neurochir Pol. 2014; 48(4): 229-235, doi: 10.1016/j.pjnns.2014.07.006, indexed in Pubmed: 25168320.

6. Li Qi, Li J, Lv F, et al. A multidetector CT angiography study of variations in the circle of Willis in a Chinese population. J Clin Neurosci. 2011; 18(3): 379-383, doi: 10.1016/j. jocn.2010.07.137, indexed in Pubmed: 21251838.

7. Pai BS, Varma RG, Kulkarni RN, et al. Microsurgical anatomy of the posterior circulation. Neurol India. 2007; 55(1): 31-41, doi: 10.4103/0028-3886.30424, indexed in Pubmed: 17272897.

8. Rai AT, Rodgers D, Williams EA, et al. Dimensions of the posterior cerebral circulation: an analysis based on advanced non-invasive imaging. J Neurointerv Surg. 2013; 5(6): 597-600, doi: 10.1136/neurintsurg-2012-010549, indexed in Pubmed: 23178224.

9. Sahni D, Jit I, Lal V. Variations and anomalies of the posterior communicating artery in Northwest Indian brains. Surg Neurol. 2007; 68(4): 449-453, doi: 10.1016/j. surneu.2006.11.047, indexed in Pubmed: 17905073.

10. Shrontz C, Dujovny M, Ausman JI, et al. Surgical anatomy of the arteries of the posterior fossa. J Neurosurg. 1986; 65(4): 540-544, doi: 10.3171/jns.1986.65.4.0540, indexed in Pubmed: 3760965.

11. van der Lugt A, Buter TC, Govaere F, et al. Accuracy of $\mathrm{CT}$ angiography in the assessment of a fetal origin of the posterior cerebral artery. Eur Radiol. 2004; 14(9): 1627-1633, doi: 10.1007/s00330-004-2333-1, indexed in Pubmed: 15127219.

12. Vitosevic F, Rasulic L, Medenica SM. Morphological characteristics of the posterior cerebral circulation: an analysis based on non-invasive imaging. Turk Neurosurg. 2019; 29(5): 625-630, doi: 10.5137/1019-5149.JTN.22661-18.1, indexed in Pubmed: 29694661.

13. Wijesinghe P, Steinbusch HWM, Shankar SK, et al. Circle of Willis abnormalities and their clinical importance in ageing brains: A cadaveric anatomical and pathological study. J Chem Neuroanat. 2020; 106: 101772, doi: 10.1016/j. jchemneu.2020.101772, indexed in Pubmed: 32165168. 